\title{
Efectos del lavado del arroz enriquecido en su contenido de selenio
}

Effect of enriched rice washing on selenium content

Paulina Silva-Trejos'

Fecha de recepción: 5 de diciembre del 2012

Fecha de aprobación: 12 de marzo del 2013

Silva-Trejos, P. Efectos del lavado del arroz enriquecido en su contenido de selenio. Tecnología en Marcha. Vol. 26, № 3. Pág 94-99 


\section{Palabras clave}

Selenio; arroz; enriquecido; alimentos; lavado; hidruros

\section{Resumen}

El arroz es un alimento básico en la dieta de los costarricenses. Alrededor del 45\% del arroz que se consume se produce localmente y el resto se importa de Estados Unidos y Argentina. En Costa Rica, el cáncer de próstata es el tumor que con mayor frecuencia se diagnostica en hombres. Las investigaciones apoyan el efecto del selenio como antioxidante en la prevención de ese tipo de cáncer. Se estima que cada persona consume 130 gramos de arroz por tiempo de alimentación. El Ministerio de Salud, ente rector en materia de salud pública, considerando que el arroz se consume en los tres tiempos de alimentación, decretó en 2006 el enriquecimiento del grano nacional e importado con $107 \mathrm{\mu g} / \mathrm{kg}$ de selenio. Es costumbre lavar el arroz antes de cocinarlo, sin seguir las recomendaciones que se incluyen en el empaque del producto. En esta investigación se estudió el efecto del lavado sobre el contenido de selenio y se determinó que disminuye entre $51 \%$ y $79 \%$ para diferentes tipos de arroz, con un procedimiento estándar de lavado. La técnica utilizada para la medición de selenio fue la espectroscopía de absorción atómica, en la modalidad de generación de hidruros, y la digestión de las muestras se realizó con ácido nítrico en horno de microondas.

\section{Key words}

Selenium; rice; enriched; foods; washing; hydride

\section{Abstract}

Rice is a common food in the diet of Costa Ricans. Currently about $45 \%$ of the rice consumed is produced locally and the rest is imported from the United States and Argentina. In Costa Rica, prostate cancer is the most frequent tumor diagnosed in men, and investigations support the antioxidant effect of selenium in the prevention of prostate cancer. Rice consumption in Costa Rica is estimated at $130 \mathrm{~g}$ for feeding time per person. The Ministry of Health, the lead agency in public health, considering that rice is consumed in the three meals, decreed in 2006 the enrichment of domestic and imported rice with $107 \mathrm{mg} / \mathrm{kg}$ of selenium. It is customary to wash the rice before cooking it, without following the recommendations included in the product package. In this investigation we determined the effect of washing on the selenium content and found that it decreases between $51 \%$ and $79 \%$ for the different types of rice, with a standard washing procedure. The technique used for measurement of selenium was atomic absorption spectroscopy with hydride generation, and digestion of the samples was performed with nitric acid in a microwave oven. 


\section{Introducción}

El arroz en uno de los alimentos básicos de consumo en la población mundial. De acuerdo con la Organización de las Naciones Unidas para la Alimentación y la Agricultura (FAO), en un documento sobre las perspectivas a plazo medio de los productos básicos agrícolas, con proyecciones al año 2010, se esperaba un incremento en la producción mundial de arroz menor al I \%, mientras que la demanda aumentará en más del 1\% anual; para los países de América Latina se espera que el consumo por habitante se incremente. El $75 \%$ de las exportaciones mundiales de arroz en 2010 se ubicaron en Asia, específicamente en Tailandia y Vietnam, y en menor proporción en Camboya, Laos y Myanmar. Se espera que la participación de China disminuya, mientras que las exportaciones de Argentina, Egipto y Uruguay aumenten considerablemente. Chinnock (Chinnock, Bethancourt, Gómez \& Meza, 1999; Consejo Regional de Cooperación Agrícola, 1997) determinó que para las familias de San Isidro de Heredia, en Costa Rica, el consumo por tiempo de alimentación por persona era de 130,3 g.

El 90 \% de la producción mundial de arroz se concentra en Asia. China e India son los productores mayoritarios, contribuyendo con $52 \%$. Brasil es el primer productor no asiático, con 2,2 \% de la producción mundial, seguido de Estados Unidos con 1,6 \%. América Latina y el Caribe, sin tomar en cuenta la producción de Brasil, representa el 2 \% de la producción mundial. En el área centroamericana y caribeña, los principales productores son Panamá, con el 31 \% de la producción de la región, Nicaragua, con 29 \% y Costa Rica con 27 \%. La producción total de arroz de Centroamérica representa el $6 \%$ de la producción total de arroz de Estados Unidos. En Centroamérica, la producción de arroz es insuficiente, por lo que se importa, siendo el principal proveedor Estados Unidos (Benavides \& Segura, 2005).

En la década de 1990, en el área centroamericana Costa Rica era el principal consumidor de arroz, con el $26 \%$ de la producción regional, seguido por Panamá con el 24 \% (Organización de las Naciones Unidas para la Agricultura y la Alimentación, 1990).

El enriquecimiento del arroz tiene como objetivo establecer en el arroz elaborado los niveles de vitamina $B$ y de minerales que se eliminan durante su preparación. Los métodos tradicionales incluyen el arroz sancochado en ácido, el arroz enriquecido con tiamina, el arroz recubierto, el arroz artificial, el arroz enriquecido con dibenzoil-tiamina y el arroz enriquecido con una mezcla enriquecida de varios nutrientes. En este último método, el arroz elaborado se remoja con una solución de ácido acético de vitaminas hidrosolubles como tiamina, riboflavina, niacina, ácido pantoténico y piridoxina; se calienta al vapor, se seca y se reviste por separado de diferentes capas de vitamina E, hierro y calcio y luego con un material de revestimiento protectivo y un colorante alimentario natural, para impedir la pérdida del nutriente con el lavado. Los niveles que se agregan de los nutrientes son los del arroz integral, y se mezcla en proporción de 1:200 con arroz elaborado. Durante el lavado ordinario se pierde un 10\% de los nutrientes y durante la cocción otro 10\% (Organización de las Naciones Unidas para la Alimentación y la Agricultura, 1996).

De acuerdo con estadísticas de la Caja Costarricense de Seguro Social publicadas en la Guía Nacional de Cáncer de Próstata, en Costa Rica este cáncer es el tumor de mayor frecuencia diagnosticado en hombres. Su incidencia se ha incrementado de 32,64 por 100000 hombres en el año 1995 a 5।,8 por 100000 hombres en 2005. De acuerdo con Gutiérrez Góngora, el 27,5 \% de los hombres en la cuarta década y el $34 \%$ en la quinta década han presentado cáncer de próstata histológico. En el caso de hombres muy jóvenes, se ha demostrado un historial familiar muy fuerte (Gutiérrez, 1998).

En un estudio realizado en Harvard, se evaluó el consumo de selenio por la concentración de este mineral en las uñas de los pies y se determinó que el riesgo de padecer cáncer de próstata era dos tercios menor en los hombres con mayores concentraciones de selenio. La dosis diaria recomendada de selenio es de 70 mg (Richardson, 2004).

En Costa Rica, en el año 2006, en una reforma al Reglamento de Enriquecimiento de Arroz, el Ministerio de Salud decreta que el arroz pilado que se utilice para el consumo humano directo debe fortificarse con ácido fólico, vitaminas del complejo $B$, vitamina $E$, selenio y zinc, que pueden provenir de mezclas con excipientes. En el caso del selenio, se requiere que se logre una concentración mínima de $105 \mu g / k g$.

La autora de este estudio validó la metodología analítica para cuantificar el selenio en diferentes 
matrices de alimentos de la canasta básica de consumo en Costa Rica, utilizando espectroscopía de absorción atómica por generación de hidruros. También ha determinado el contenido de selenio en alimentos prioritarios de la dieta del costarricense (Silva Trejos, 20 I I).

En esta investigación se busca determinar el efecto que tiene el lavado sobre el contenido de selenio del arroz fortificado que se consume en Costa Rica.

\section{Materiales y métodos}

La muestra de laboratorio se obtuvo a partir del empaque comercial de $2 \mathrm{~kg}$ de arroz. Antes de abrirlo, se homogenizó manualmente. Por cuarteo del contenido total, se obtuvo una muestra de laboratorio de $200 \mathrm{~g}$, de donde por duplicado se determinó la humedad y se obtuvieron por duplicado muestras para digestión en horno de microondas con acido nítrico concentrado.

Para determinar el efecto del lavado y considerando que los volúmenes y los tiempos de lavado utilizados por las personas no están estandarizados, se tomaron $25 \mathrm{~g}$ de arroz del empaque comercial y se lavaron con $100 \mathrm{~mL}$ de agua desionizada por espacio de 5 minutos con agitación, posteriormente se filtró y se cocinó con un volumen de agua desionizada por 4 minutos en horno de microondas a una potencia de 10. El arroz cocido se liofilizó y se le determinó la humedad, antes de proceder a la digestión en horno de microondas para cuantificar el contenido de selenio.

Se determinó la humedad de la muestra de arroz crudo y de la misma muestra de arroz cocido. El arroz cocido se liofilizó y se tomaron muestras por duplicado para determinar la humedad. Se digirieron por duplicado muestras del arroz crudo y del arroz liofilizado, a las que se les determinó el contenido de selenio. Los resultados se corrigieron con los resultados de humedad para hacer la comparación sobre base seca. Para la determinación de la humedad se aplicó el método oficial AOAC 925.09.

Las lecturas de selenio se realizaron en el espectrómetro Varian modelo 220 Fast Sequential, provisto de una lámpara de selenio y llama aire acetileno. Se trabajó a una longitud de onda igual a 196 nm y una corriente de $10 \mathrm{~mA}$ y corrector de radiación de fondo con lámpara de deuterio.
Para la preparación de la curva de calibración se utilizó una disolución patrón de selenio J.T.Baker trazable a la NISTß con una concentración de (I000 \pm I) $\mathrm{mg} / \mathrm{L}$ al $5 \%$ en HNO3. Los patrones para la curva de calibración se prepararon en el ámbito de ( I-9) $\mu g / L$, y se trataron con $\mathrm{HCl}$ concentrado para garantizar que cuantitativamente todo el selenio se encuentre en el estado de oxidación +4 .

La digestión de las muestras se realizó en un horno de microondas Millestone, modelo ETHOS PLUS. Se midieron masas de 0,5 g a las cuales se les adicionaron 5,0 mL de $\mathrm{HNO} 3$ al $65 \%$.La muestra digerida se calentó con $\mathrm{HCl}$ concentrado por 15 minutos a (70-90) ${ }^{\circ} \mathrm{C}$, para garantizar que todo el selenio se encuentrara en el estado de oxidación +4 (Varian Instruments at Work, 1983).

El control de calidad de la respuesta del espectrómetro se realizó con una disolución de concentración intermedia de la curva de calibración, preparada a partir de una disolución de selenio trazable. La veracidad se evaluó analizando por duplicado muestras de material de referencia certificado Rice Flour 1568 NIST® cada veinte muestras de arroz analizadas. La curva de calibración se preparó para cada sesión de lectura de muestras y se preparó un ensayo en blanco cada veinte muestras de arroz analizadas.

\section{Resultados}

Se determinó el contenido de humedad para las muestras de arroz cocidas y liofilizadas con el método oficial AOAC 925.09, con la finalidad de comparar los contenidos de selenio referidos a la muestra de arroz seca. En el cuadro I se presentan los resultados obtenidos.

En la figura I se presenta la curva de calibración utilizada para la cuantificación de las muestras y el material de referencia certificado.

Los límites de detección y de cuantificación, según

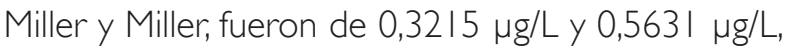
respectivamente (Miller \& Miller, 2002).

En en el cuadro 2 se presentan los resultados como porcentaje de pérdida de selenio obtenidos para las muestras de arroz crudo y lavado y cocido, ambos resultados referidos a base seca según los resultados de humedad obtenidos. 
Cuadro I. Humedad para las muestras de arroz cocidas y liofilizadas.

\begin{tabular}{|c|c|c|}
\hline Muestra de arroz & Humedad muestra fresca \% & Humedad muestra liofilizada \% \\
\hline A-025 & 58,0 & 0,8 \\
\hline A-028 & 62,9 & 0,6 \\
\hline A-030 & 69,1 & 1,8 \\
\hline A-031 & 55,4 & 1,6 \\
\hline A-032 & 59,2 & 1,8 \\
\hline A-034 & 41,4 & 1,9 \\
\hline
\end{tabular}

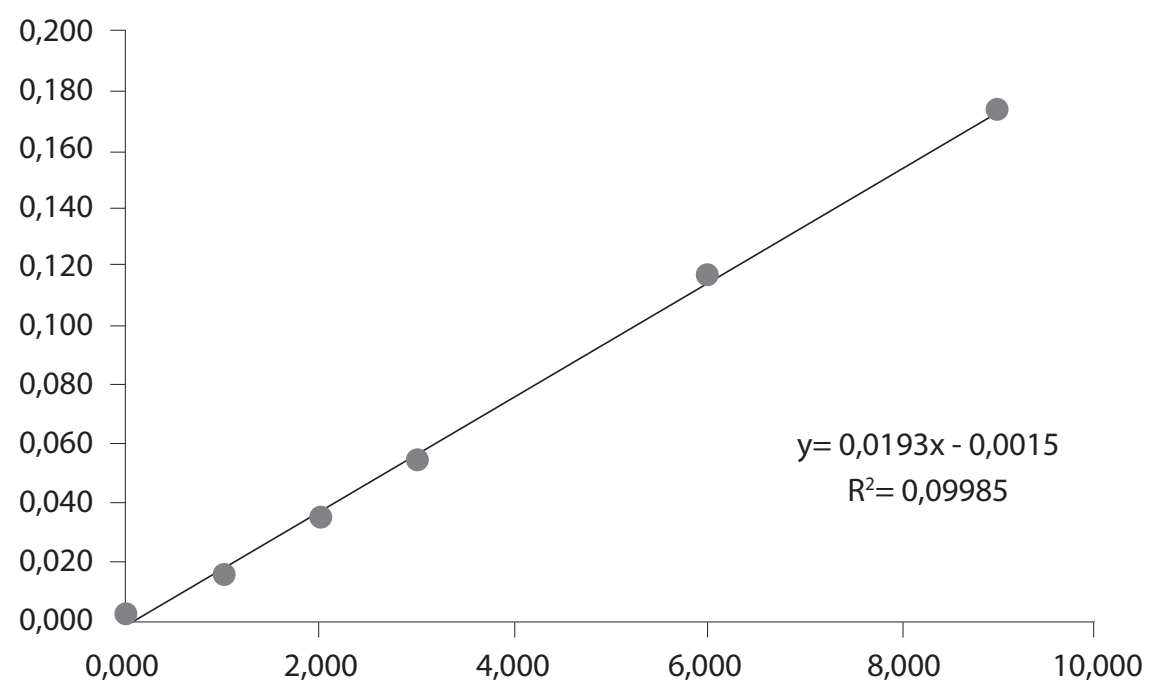

Figura I. Curva de calibracón para la cuantificación de las muestras y el material de referencia certificado.

Fuente: P. Silva Trejos

\section{Discusión}

Un estudio de la FAO evidencia que el lavado del arroz previo a la cocción elimina parte de sus nutrientes, entre ellos proteínas, minerales y vitaminas hidrosolubles. Durante la cocción, el volumen de agua utilizado determina una nueva pérdida de nutrientes. Se registran pérdidas en los nutrientes por efecto del lavado y la cocción en arroz pulido al $90 \%$ para hierro y calcio de $23 \%$ y $21 \%$ respectivamente, y para tiamina, riboflavina y niacina de $51 \%, 35 \%$ y 55 \% respectivamente (Organización de las Naciones Unidas para la Agricultura y la Alimentación, 1990).

Los resultados obtenidos en esta investigación permiten determinar que al aplicarse un procedimiento de lavado y cocción normalizado a diferentes clases de arroz, se aprecia en todos los casos una pérdida significativa en el contenido de selenio, que oscila entre $51 \%$ y $79 \%$. Para el arroz precocido se observa que al aumentar el porcentaje de grano entero se incrementa el porcentaje de pérdida de selenio; mientras que el porcentaje más alto de pérdida lo obtuvo el arroz blanco.

En los empaques de las muestras de arroz consideradas en este proyecto se observaron las siguientes declaraciones: "Ahora se puede lavar sin perder nutrientes" y "No necesita lavarse", siendo ambas contradictorias para el consumidor. Por otro lado, los resultados experimentales demuestran que sí se presentan pérdidas considerables en el arroz lavado, por lo que se deberían unificar las recomendaciones al consumidor para indicar con claridad que no debe lavarse para evitar la pérdida de los nutrientes adicionados. 
Cuadro 2. Porcentaje de pérdida de selenio.

\begin{tabular}{|c|c|c|c|}
\hline Muestra de arroz & $\begin{array}{c}\mu g \text { de Se/kg en arroz crudo } \\
\text { sobre base seca }\end{array}$ & $\begin{array}{c}\mu g \text { de Se/kg en arroz lavado y } \\
\text { cocido sobre base seca }\end{array}$ & \% de pérdida \\
\hline A, precocido al 94\% & 174,9 & 50,4 & 71 \\
\hline B, precocido al 98\% & 80,9 & 29,7 & 63 \\
\hline C, precocido & 180,6 & 51,3 & 51 \\
\hline D, blanco al 91 \% & 132,7 & 79,4 & 79 \\
\hline
\end{tabular}

\section{Agradecimientos}

La autora agradece a la Vicerrectoría de Investigación de la Universidad de Costa Rica, por el apoyo económico brindado para realizar esta investigación.

\section{Bibliografía}

Chinnock, A., Bethancourt, V., Gómez, A. \& Meza, M. (1999). Comparación entre dos métodos para medir el consumo de alimentos en un área rural costarricense. Rev. Costarric. Salud Pública, v. 8, n. 15, dic.

Consejo Regional de Cooperación Agrícola. (1997). Situación actual del comercio y la producción actual de arroz del Sudeste Asiático, sus implicaciones para Centroamérica.

Gutiérrez G., J. (1998). Cáncer de la próstata. San José: Editorial Universidad de Costa Rica.
Miller J., N. \& Miller, J.C. (2002). Estadística y Quimiometría para Química Analítica. 4 ed. Madrid: Prentice Hall.

Organización de las Naciones Unidas para la Alimentación y la Agricultura. (1996). El Arroz en la Nutrición Humana. Roma.

Organización de las Naciones Unidas para la Agricultura y la Alimentación. (1990). Utilización de Alimentos Tropicales: Cereales.

Richardson, M.S. (2004). Enciclopedia de la Salud. Barcelona: Amat Editorial.

Benavides, H. \& Segura, O. (2005). El entorno internacional del sector arrocero centroamericano. 4 ed. IICA.

Silva Trejos, P. (20I I).Validación de la metodología analítica para cuantificación de selenio en alimentos de la canasta básica del costarricense. Rev. Costarric. Salud Pública, v. 20, n. I, jun.

Varian Instruments at Work. (1983). An automatic Vapor Generation Accessory for Atomic Absorption Analysis. Number AA-38. 